EDITORIAL

\title{
¿Innovaciones educativas o pedagógicas?
}

$\mathrm{L}$

a velocidad de los cambios producidos en el mundo en las últimas décadas ha privilegiado diversas formas de transformación en las maneras de enseñar y aprender;

lestos cambios requieren procesos acelerados de pensar todas las realidades y realizar sus respectivas transformaciones. La innovación se hace parte central de este cambio, pues es la única posibilidad de conexión con un mundo cada más vertiginoso; curiosamente, como ha sucedido con muchos conceptos y categorías utilizados hoy en el ámbito educativo, la innovación emergió en el ámbito de la economía y de la empresa; los economistas y los empresarios comenzaron a utilizar el concepto para alterar los ciclos productivos y lograr desequilibrios que convirtieran los procesos de producción en dinámicos y eficientes. En otro momento la innovación se enarboló para evitar los riesgos de la innovación espontánea, es decir, que las alteraciones no tuviesen control, y se opta por producir la innovación a través de la optimización de los procesos de gestión del conocimiento con el fin de lograr ciclos sostenidos y constantes de innovación. En una etapa posterior se regulariza la producción de la innovación a través de los modelos de magnament y la gestión.

Para el caso de la educación, el término innovación se ha tornado polisémico, complejo y, a veces, hasta confuso o ambiguo; generalmente se utiliza para nombrar situaciones de cambio o transformación de las realidades educativas; algunas veces, para referirse a las reformas que desde los entes gubernamentales se realizan; en otras ocasiones, para mostrar la implementación de lo que hoy conocemos como "modelo pedagógico", es decir, los cambios en la perspectiva de la escuela, e incluso de la universidad, y, por último, se ha denominado innovación a los cambios y adaptaciones en el componente técnico y didáctico de la práctica que realiza el maestro en el aula. 
El cambio y la transformación se han convertido en categorías e imaginarios que movilizan toda la realidad y hacen que el cuestionamiento sobre la verdad y la razón sea cada vez mayor, y surjan los discursos de la incertidumbre, la indeterminación y la relatividad del saber; esto hace que los sistemas educativos se enfrenten a estos cambios, y los asuman de manera crítica o se dejen vencer por la fuerza de la novedad. Estos cambios se manifiestan en la escuela y la educación formal en el desplazamiento de la enseñanza al aprendizaje (aprender a aprender), en las transformaciones de la cultura escolar (nuevos actores, protagonistas y discursos) y en los modelos de gestión y administración de las instituciones educativas; formas flexibles del currículo, formación permanente y función docente suceden en el tiempo y con variedad en la intensidad como en la intencionalidad; la innovación representa la llegada de ideas nuevas y creativas, y en ese sentido una posibilidad de comprender e intervenir el sistema o una forma de adaptación y servilismo a éste.

La Maestría en Educación de la UPTC, a partir de su primera cohorte, ha logrado consolidar un grupo de estudiantes y profesores que han dedicado sus esfuerzos investigativos a caracterizar y poner en funcionamiento el concepto de innovación pedagógica, lo que ha permitido dinamizar trabajos en distintas instituciones educativas y poblaciones con el convencimiento de que la innovación debe superar el ámbito generalizado del cambiar por cambiar o por moda o por imposición. Adicionalmente, se ha ido tomando conciencia de que la educación colombiana presenta una situación de crisis, como todo el conjunto de la sociedad, y de que es necesario introducir cambios que de alguna manera puedan contribuir a que los niños, niñas y jóvenes que participan del sistema educativo colombiano puedan encontrar en él respuestas a sus profundas aspiraciones y procesos de constitución como sujetos contemporáneos. Por eso la investigación y puesta en marcha de innovaciones se ubica más en la perspectiva pedagógica, es decir, que la innovación no es un simple aparato técnico-instrumental, sino que se convierte, en un primer momento, en un proceso práctico-reflexivo, es decir, en la posibilidad de acción del maestro, profesor, docente, sobre un campo de conocimiento y sobre todo en relación con un espacio de creatividad y de interacción. Otro elemento hace referencia a la doble posibilidad de la innovación: crítica y creativa. Crítica, en tres dimensiones: en primer lugar, el reconocimiento de los límites de los discursos y las prácticas para comprender y totalizar la realidad, en este caso las prácticas y los discursos escolares; en segundo lugar, la crítica permite desnaturalizar conceptos y hábitos, es decir, impide asumir prácticas o usos sociales sin su revisión y cuestionamiento, y por último, la crítica afirma el sentido de la pregunta, la 
interrogación constante, el cuestionamiento de la verdad; en definitiva, la crítica nos permite pensar qué y para qué hacemos lo que hacemos, decimos o pensamos. La dimensión creativa de la innovación ocupa el espacio que permite producir sentidos, derroteros y líneas que permitan que los sujetos involucrados en ella se ubiquen y desplacen en los mapas de subjetivación que la innovación impulsa. Este segundo número de la revista Praxis \& Saber tiene como eje central de discusión las innovaciones pedagógicas desde diferentes perspectivas y ámbitos de aplicación.

El artículo que inaugura esta edición presenta una experiencia personal, en el texto denominado Educación crítica: historia de una resistencia en palabra y acción, de uno de los más grandes pedagogos críticos del mundo contemporáneo: el profesor Michel Apple; en él se expresa que la pedagogía crítica no puede quedarse en el discurso o la conversación académica, sino que debe trascender hasta llegar a la acción y conectarse con los movimientos sociales y educativos que los sujetos encarnan. Los siguientes artículos presentan una serie de resultados de investigación de orden teórico y práctico a partir de innovaciones pedagógicas en relación con la magia, el cine, las artes plásticas, las nuevas tecnologías y la enseñanza de las matemáticas.

La profesora María Teresa Suárez Vaca, directora del Jardín Infantil de la Uptc y estudiante de la Maestría en Educación, en su artículo, titulado La magia y una nueva relación pedagógica con la infancia, plantea las relaciones entre conocimiento y magia y su utilización como campo de acceso y producción de conocimiento. Enseguida, dos artículos abordan la relación cine y pedagogía: los profesores Angélica Osorio y Víctor Manuel Rodríguez, de la Universidad Pedagógica Nacional, presentan resultados del proyecto de investigación titulado "El cine como posibilidad de pensamiento desde la pedagogía: una mirada a la formación de maestros", en su artículo Cine y pedagogía: reflexiones a propósito de la formación de maestros, donde ubican la formación en una acepción de experiencia, y la innovación pedagógica como campo de problematización; y la profesora Luz Marina Nana Rodríguez, profesora de la Uptc, presenta los resultados de su tesis de maestría, en su artículo titulado El cine, una estrategia para el desarrollo del pensamiento, que tuvo como objetivo principal diseñar y aplicar una estrategia basada en el cine que permita hacer rupturas con la imagen dogmática del pensamiento en los estudiantes universitarios.

La innovación educativa ha estado muy vinculada en algunos ámbitos teóricos y de intervención con la implementación de nuevas tecnologías de la información y la comunicación; en este sentido, la profesora Dora Lidia Orjuela Forero presenta el trabajo titulado Acercamiento a la integración curricular de las TIC, que presenta inicialmente una revisión teórica sobre formas y 
maneras de integración, y luego expone una experiencia concreta en una institución educativa de Boyacá.

Los artículos siguientes presentan experiencias y reflexiones sobre la innovación y las didácticas de la artes. Carlos Báez, profesor de una institución educativa de educación básica y media en el municipio de Ráquira, Boyacá, y estudiante de la Maestría en Educación de la Uptc, con su trabajo titulado La escuela emergente a través de la práctica artística, la experiencia y la subjetividad, hace énfasis en el lugar determinante de la educación artística en las instituciones educativas y del desarrollo del pensamiento artístico por parte de los docentes del área. En el mismo sentido, el profesor Héctor Monsalve, docente en una institución educativa del municipio de Paipa, Boyacá, y estudiante de la Maestría en Educación de la Uptc, con su artículo Momentos del dibujo: un acercamiento a la condición escolar, presenta una nueva perspectiva para orientar el dibujo infantil en la lógica de la creación.

El lector de Praxis \& Saber puede acceder al artículo La comunicación: eje en la clase de matemáticas, de los profesores Alfonso Jiménez Espinosa, Nury Yolanda Suárez Ávila y Sandra María Galindo Mendoza, del Grupo de Investigación Pirámide, en el cual se presentan aspectos relacionados con el aprendizaje y la enseñanza de las matemáticas, teniendo como foco central la comunicación, entendida como un proceso de interacción social.

Luego de este bloque de artículos dedicado a la innovación pedagógica, el profesor Óscar Saldarriaga Vélez presenta los resultados de un proyecto de investigación sobre las pruebas comprender, en su texto titulado Evaluación de la construcción del conocimiento social. Análisis de los sistemas de sentido en alumnos de $5 .^{\circ}$ y $9 .^{\circ}$ grados en colegios de Bogotá. 